\title{
Projekt DEAL:
}

\section{Open Access-Publizieren für Deutschland}

DOI: $10.1007 / \mathrm{s} 12268-020-1339-9$

(C) Springer-Verlag 2020

Mit der deutschlandweiten DEAL-Vereinbarung können seit Januar 2020 rund 1.000 deutsche Universitäten und Forschungseinrichtungen an der Open Access-Vereinbarung teilnehmen, die zwischen den von Projekt DEAL vertretenen Institutionen und dem Wissenschaftsverlag Springer Nature getroffen wurde.

Dieser Vereinbarung nach können Wissenschaftler aus den angeschlossenen Einrichtungen in den etwa 1.900 Hybrid-Zeitschriften * von Springer Nature kostenfrei Open Access (OA) veröffentlichen. Das gilt auch für BIOspektrum.

Der Vertrag regelt darüber hinaus, dass die Forscher und Studierenden der angeschlossenen Institutionen die Zeitschriften-Inhalte des Verlags auch kostenfrei nutzen können. (Publish \& Read, abgekürzt PAR).

Der komplette Vertragstext wird auf der Projekt DEAL-Webseite veröffentlicht. Die Vereinbarung gilt zunächst für den Zeitraum bis 2022, mit der Option einer Verlängerung bis 2023. Die Zeitschriften der Marke Nature sind nicht Bestandteil des DEAL-Abkommens. Weitere Informationen über Projekt DEAL finden Sie hier: www.projekt-deal.de

Fest definierte Typen von Artikeln können im Rahmen des DEAL-Abkommens Open Access publiziert werden - kostenfrei für Autor/innen, sofern diese einer der teilnehmenden Institutionen angehören.

Dies gilt für BIOspektrum-Artikel, bei welchen die folgenden Voraussetzungen erfüllt sind:

- Berechtigte Artikeltypen in BIOspektrum sind die Fachartikel aus der Rubrik Wissenschaft sowie die Special- und die Bio-

\footnotetext{
* Hybrid-Zeitschriften publizieren neben herkömmlich veröffentlichten Subskriptionsartikeln zusätzlich auch Open Access-Beiträge.
}

technologie-Artikel (Review Papers), unsere Editorials (Editorial Notes), die Buchrezensionen (Book Reviews) und einige der KKK-Artikel (Reports).

- Der/die korrespondierende Autor/in des Artikels ist an einer der teilnehmenden Institutionen und hat dies im zu publizierenden Manuskript kenntlich gemacht (Korrespondenzadresse).

- Die Institution, der/die korrespondierende Autor/in angeschlossen ist, hat nach Prüfung die Freigabe erteilt, dass der Artikel den formalen Anforderungen zur Open Access-Publikation entspricht. Das ist wichtig, denn diese Institution ist später der Rechnungsempfänger.

Details zu den oben genannten Punkten finden Sie unter diesem Link: $w w w . s p r i n g e r$ nature.com/gp/open-research/institutionalagreements/oaforgermany-de

Seit 2015 hat Springer Nature bereits weitreichende Erfahrungen mit transformativen OA-Vereinbarungen in vorwiegend europäischen Ländern gesammelt. Insgesamt gibt es derzeit zehn solcher Vereinbarungen, die die jeweiligen Anforderungen individuell regeln.

Einzelheiten zu den Vereinbarungen mit den Niederlanden, Österreich, Schweden, Norwegen, Finnland, Ungarn, Großbritannien, Polen, Qatar und der Max-PlanckGesellschaft sind hier nachzulesen: www.springer.com/de/open-access/springeropen-choice/springer-compact

Eine globale Analyse hat gezeigt, wie sich Open Access auf die Sichtbarkeit der publizierten Inhalte sowie die Artikel-Downloadzahlen und Zitierraten auswirkt (Assessing the open access effect for hybrid journals: www.springernature.com/gp/open-research/ about/oa-effect-hybrid). Open Access-Artikel werden im Vergleich zu Artikeln, die hinter einer Bezahlschranke sind,

- in den akademischen Institutionen im Durchschnitt $1,6 \mathrm{mal}$ so oft herunterge- laden; über alle Interessenten betrachtet erhöhen sich die Downloadzahlen um Faktor 4

- durchschnittlich 1,6mal mehr zitiert;

- erhalten im Schnitt 2,4mal mehr Aufmerksamkeit im Internet und in den sozialen Netzwerken;

- und werden 1,9mal häufiger in Nachrichtenmeldungen erwähnt.

Wir freuen uns, mit BIOspektrum Teil dieses transformativen Prozesses hin zu Open Access zu sein und sind gespannt auf die gemeinsamen Erfahrungen.

Ihre BIOspektrum-Redaktion

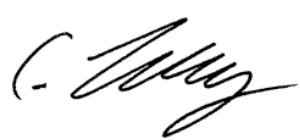

Claudia Ludy

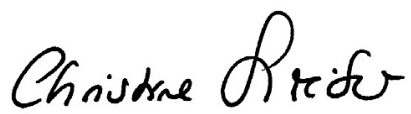

Christine Schreiber

\section{Brigitte Irageser}

Brigitte Trageser

Korrespondenzadresse:

Dr. Christine Schreiber Springer-Verlag $\mathrm{GmbH}$, ein Teil von Springer Nature Tiergartenstraße 17 D - 69121 Heidelberg Tel.: 06221-487-8043 biospektrum@springer.com www.springer.com www.biospektrum.de 